\title{
A New Four-Dimensional Autonomous Hyperchaotic System and the Synchronization of Different Chaotic Systems by Using Fast Terminal Sliding Mode Control
}

\author{
Baojie Zhang ${ }^{1,2}$ and Hongxing $\mathrm{Li}^{1}$ \\ ${ }^{1}$ Faculty of Electronic Information and Electrical Engineering, Dalian University of Technology, Dalian 116024, China \\ ${ }^{2}$ School of Mathematics and Information Science, Qujing Normal University, Qujing 655011, China \\ Correspondence should be addressed to Baojie Zhang; qjupk123@mail.dlut.edu.cn
}

Received 24 September 2013; Revised 28 November 2013; Accepted 28 November 2013

Academic Editor: Tomasz Kapitaniak

Copyright (C) 2013 B. Zhang and H. Li. This is an open access article distributed under the Creative Commons Attribution License, which permits unrestricted use, distribution, and reproduction in any medium, provided the original work is properly cited.

This paper presents a new continuous-time four-dimensional autonomous system based on Lorenz system. We analyze the dissipation, equilibrium, and Lyapunov exponents of the system. Lyapunov exponent spectrum demonstrates that the system possesses rich dynamic behaviors if the parameters of the system vary. In a large range of parameters, the system is hyperchaotic. By using fast terminal sliding mode control method, the synchronization of two different chaotic systems is studied. Synchronization between the new system and hyperchaotic Chen system with noise perturbation is illustrated. Simulation results verify the effectiveness of the proposed method.

\section{Introduction}

Since Lorenz first discovered the chaotic attractor [1] in 1963, many new chaotic systems have been found, and there is a lot of interesting work on the study of chaotic systems, such as Rossler system [2], Genesio system [3], Chen system [4], Lü system [5, 6], Liu system [7], and Qi system [8]. These chaotic systems have unique positive Lyapunov exponent. Positive Lyapunov exponent is an important character of chaotic system, and it is a value that describes the sensitive dependence on initial conditions. More positive Lyapunov exponents mean that the system is of much more chaos. Rossler defined that a system with at least two positive Lyapunov exponents is hyperchaotic in 1979. Meanwhile, he presented the first hyperchaotic system-hyperchaotic Rossler system [9]. The dynamic behavior of hyperchaotic system is more complicated and unpredictable. With these characters, it is important to use hyperchaotic system in engineering, especially in secure communication. Over the past decades, many hyperchaotic systems have been introduced, such as hyperchaotic Chen system [10] by Chen and Dong, hyperchaotic Chen system [11] by Li et al., hyperchaotic Lü system by Chen et al. [12], hyperchaotic Nikolov system [13] by Nikolov and Clodong, hyperchaotic Lorenz system [14] by $\mathrm{X}$. Wang and $\mathrm{M}$. Wang, and hyperchaotic Lorenz system [15] by Barboza. This paper presents a new hyperchaotic system by adding a state variable $w$ and analyze the new system's dissipation, the existence of the attractor, the stability of the equilibriums, and the dynamic behavior as a parameter varies.

Since Pecora and Carroll observed the synchronization of chaotic systems [16], there have been many efforts on the scheme of synchronization and a variety of approaches have been proposed for synchronization of chaotic systems, such as linear and nonlinear feedback synchronization [17, 18], adaptive synchronization [19-21], active synchronization [22] and sliding mode synchronization [23, 24]. For the synchronization of different chaotic systems that exists extensively, it is more challenging to study the synchronization of two different chaotic systems than to research the identical ones. It is very useful in secure communication. Here we utilize terminal sliding mode method to synchronize two different chaotic systems. Synchronization of the new hyperchaotic 
system and the hyperchaotic Chen system illustrated the presented approach.

The structure of this paper is organized as follows. In Section 2, the design of the new hyperchaotic system is introduced. The dynamic behaviors are analyzed. In Section 3, the scheme of terminal sliding mode synchronization between two different chaotic systems with disturbances is proposed. In Section 4, numerical simulations of the synchronization between the new hyperchaotic system and the hyperchaotic Chen system verify the effectiveness of the scheme. Finally, conclusions are given in Section 5.

\section{The Design of a New Hyperchaotic System}

Lorenz system is a paradigm of chaos given by

$$
\begin{gathered}
\dot{x}=a(-x+y), \\
\dot{y}=b x-y-x z, \\
\dot{z}=-c z+x y,
\end{gathered}
$$

where $x, y$, and $z$ are state variables and $a, b$, and $c$ are parameters. Here $a=10, b=28$, and $c=8 / 3$. Adding a new state variable $w$ to the second equation with the change rate of $w$ being $d w-x z$ and adding $0.67 w$ to the third equation of Lorenz system, we get a system as follows:

$$
\begin{gathered}
\dot{x}=a(-x+y), \\
\dot{y}=b x-y+w-x z, \\
\dot{z}=-c z+0.67 w+x y, \\
\dot{w}=d w-x z .
\end{gathered}
$$

When $a=31, b=54, c=13$, and $d=1.8$, the Lyapunov exponents are $\mathrm{LE}_{1}=1.156884, \mathrm{LE}_{2}=0.720394$, $\mathrm{LE}_{3}=0.026702$ and $\mathrm{LE}_{4}=-44.999849 . \mathrm{LE}_{3}=0.026702$ is close to 0 . Obviously, with these parameters, the system is hyperchaotic. The phase portraits are shown in Figure 1.

2.1. Dissipativity and Existence of Attractor. For the system (2), we have

$$
\begin{aligned}
\nabla V & =\frac{\partial \dot{x}}{x}+\frac{\partial \dot{y}}{y}+\frac{\partial \dot{z}}{z}+\frac{\partial \dot{w}}{w} \\
& =-a-1-c+d
\end{aligned}
$$

If $\nabla V=-a-1-c+d<0$, the system is dissipative. When $a=31, b=54, c=13, d=1.8$, and $\nabla V=-43.2$, the system (2) is dissipative and it converges with the rate $e^{-43.2 t}$. That is to say, when $t \rightarrow \infty$, each volume containing a trajectory contracts to vacancy and the trajectory is confined in an attractor.

2.2. Equilibriums and Stability. By solving the equilibrium equation of system (2), we know that the system has three equilibria $O_{1}(0,0,0,0)$, $\mathrm{O}_{2}(-67.3912,-67.3912,119.25,-4464.6692)$,
$\mathrm{O}_{3}(23.0037,23.0037,119.25,1523.9974)$. By linearizing system (2), we obtain the Jacobian

$$
A=\left[\begin{array}{cccc}
-a & a & 0 & 0 \\
b-z & -1 & -x & 1 \\
y & x & -c & 0.67 \\
-z & 0 & -x & d
\end{array}\right]
$$

From the Jacobian (4), we know that the eigenvalues at $\mathrm{O}_{1}$ are $\lambda_{\mathrm{O} 11}=-59.5775, \lambda_{\mathrm{O} 12}=27.5775, \lambda_{\mathrm{O} 13}=-13.0000$, and $\lambda_{\mathrm{O} 14}=1.8000$; the eigenvalues at $\mathrm{O}_{2}$ are $\lambda_{\mathrm{O} 21}=0.2302+$ $83.2288 i, \lambda_{\mathrm{O} 22}=0.2302-83.2288 i, \lambda_{\mathrm{O} 23}=-44.1544$, and $\lambda_{\mathrm{O} 24}=0.4939$; the eigenvalues at $\mathrm{O}_{3}$ are $\lambda_{\mathrm{O} 31}=-9.7166+$ $48.9668 i, \lambda_{\mathrm{O} 32}=-9.7166-48.9668 i, \lambda_{\mathrm{O} 33}=-24.6077$ and $\lambda_{\mathrm{O} 34}=0.8409$. Because each equilibrium has at least an eigenvalue with positive real part, the three equilibriums are all unstable.

2.3. Bifurcation and Lyapunov Exponent Spectrum. Without loss of generality, we fix the parameters $a, b$, and $c$. Let $a=31$, $b=54$, and $d=1.8$. The bifurcation diagram in $x$-direction during $c$ varies in the interval $[1,20]$ is shown in Figure 2.

The bifurcation diagrams in $y$-direction, $z$-direction, and $w$-direction are almost the same as Figure 2 . The system dynamic behavior can be defined by one component.

The Lyapunov exponents are shown in Figures 3 and 4.

In Figures 3 and 4, we observe that there are two positive Lyapunov exponents over a large range of parameters c. Two positive Lyapunov exponents imply that system (2) is hyperchaotic. As we all know, the signs of Lyapunov exponents characterize different dynamic behaviors of the system. Specifically, when $c \in[1,1.2864], c \in$ (4.0553, 4.4372], $c \in(6.5377,7.6834], c \in(8.4472,8.6382]$, and $c \in(9.7839,10.6432]$, one Lyapunov exponent is zero and the other three are negative, and system (2) is periodic; when $c \in(1.2864,1.7638], c \in(4.4372,4.6281]$, $c \in(7.6834,7.8744]$, two Lyapunov exponents are zero and the other two are negative, it is quasiperiodic; when $c \in$ $(1.7638,2.2412], c \in(5.1055,6.5377], c \in(7.8744,8.4472]$, $c \in(8.6382,9.2111]$ and $c \in(15.8945,20]$, only one Lyapunov exponent is positive, it is chaotic; when $c \in$ (2.2412, 4.0553], $c \in(4.6281,5.1055], c \in(9.2111,9.7839]$ and $c \in(10.6432,15.8945]$, two Lyapunov exponents are positive, it is hyperchaotic. To observe the phase portraits of system (2), we get four typical attractors of the system by selecting $c=1.1, c=7.87, c=6$ and $c=2.8$. Their phase portraits are shown in Figures 5, 6, 7, and 8, respectively.

\section{Fast Terminal Sliding Synchronization between Two Different Chaotic Systems}

Terminal sliding mode control is an effective approach for chaotic synchronization $[25,26]$. In this section, we present the fast terminal sliding synchronization method to synchronize two different chaoses with disturbance. Let $\dot{x}=f(x)$ and $\dot{y}=g(y)+\delta(t)+u$ be drive system and response system, respectively. $x, y \in R^{n}$ are state variables. $\delta(t) \in R^{n}$ is the disturbance and $u \in R^{n}$ is the controller. The state error 


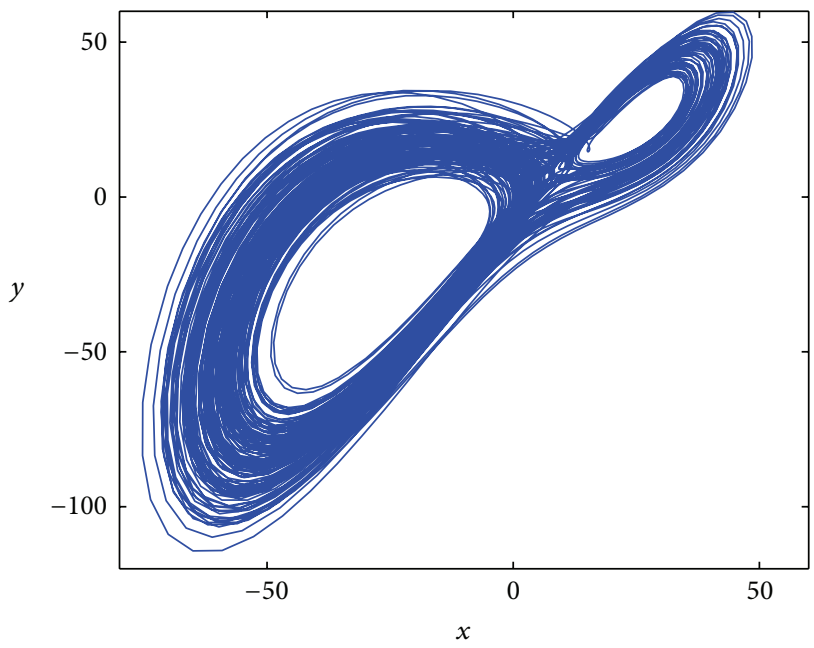

(a)

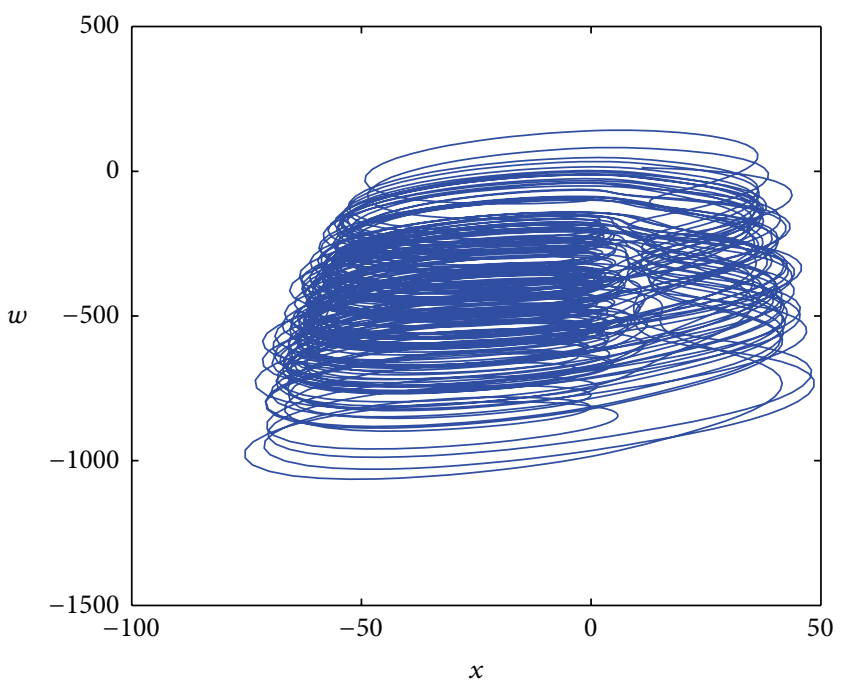

(c)

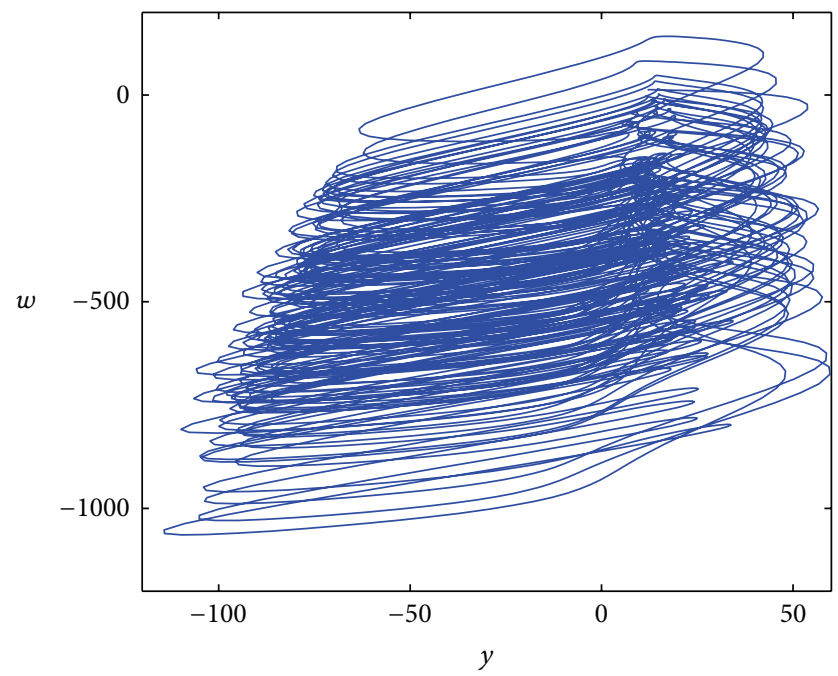

(e)

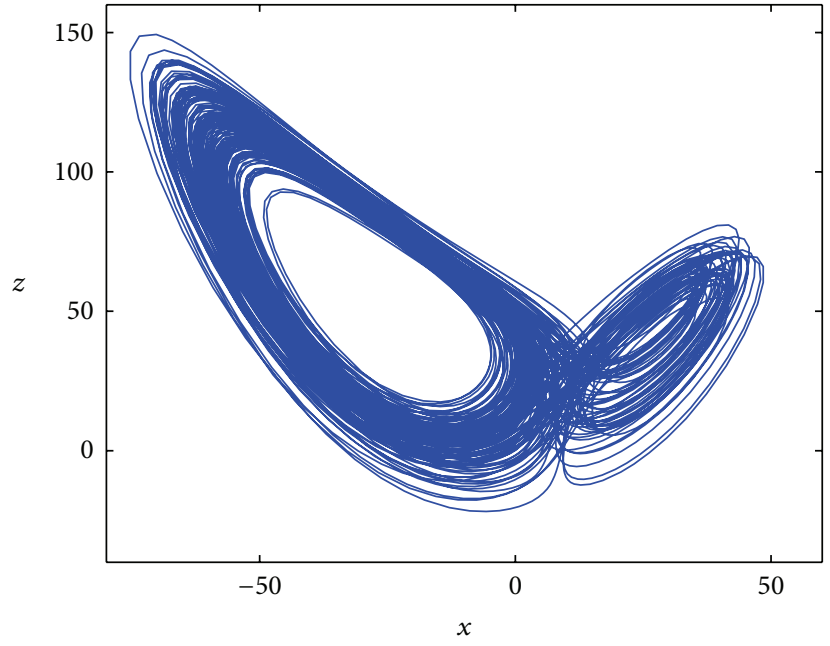

(b)

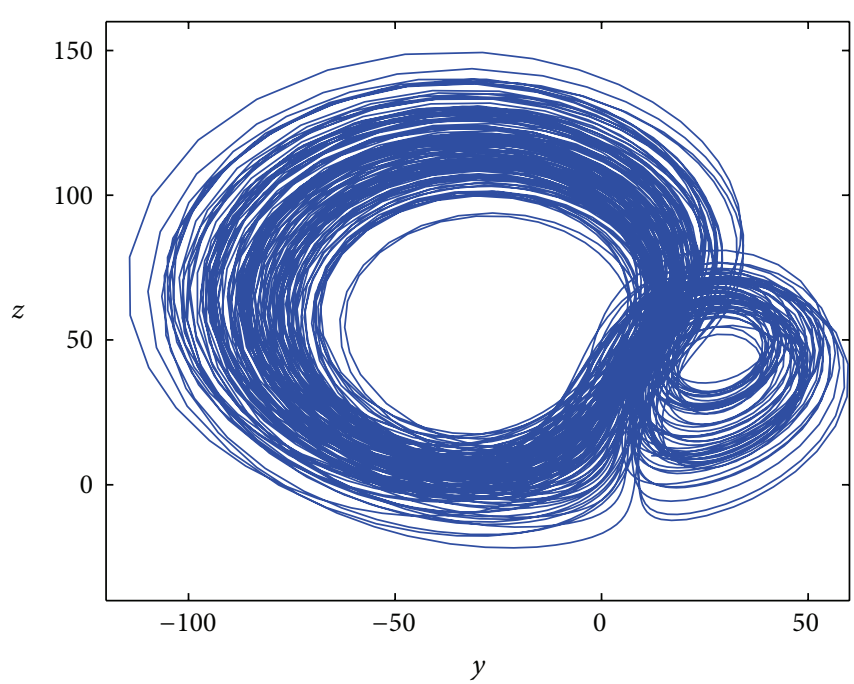

(d)

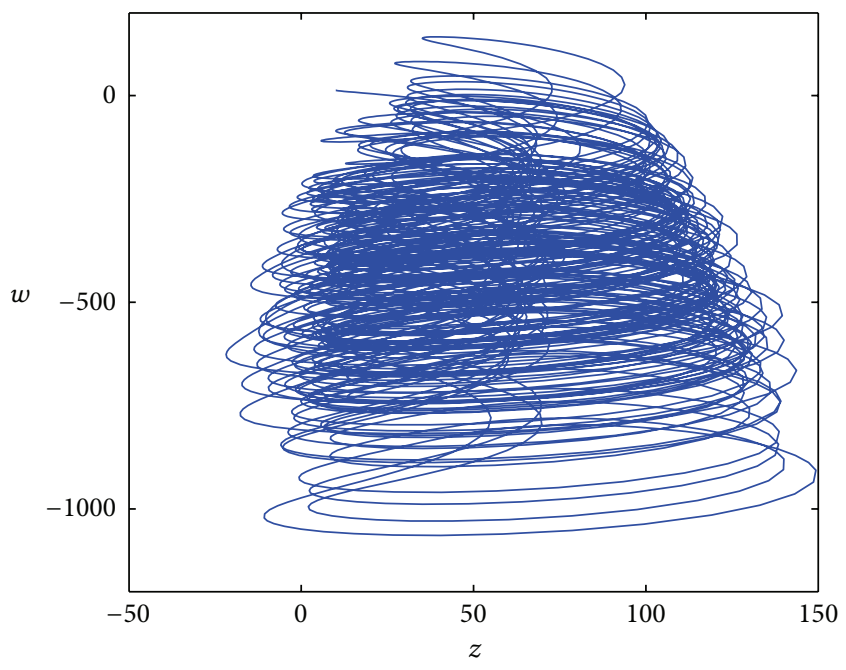

(f)

FIGURE 1: The phase portrait of hyperchaotic system. 


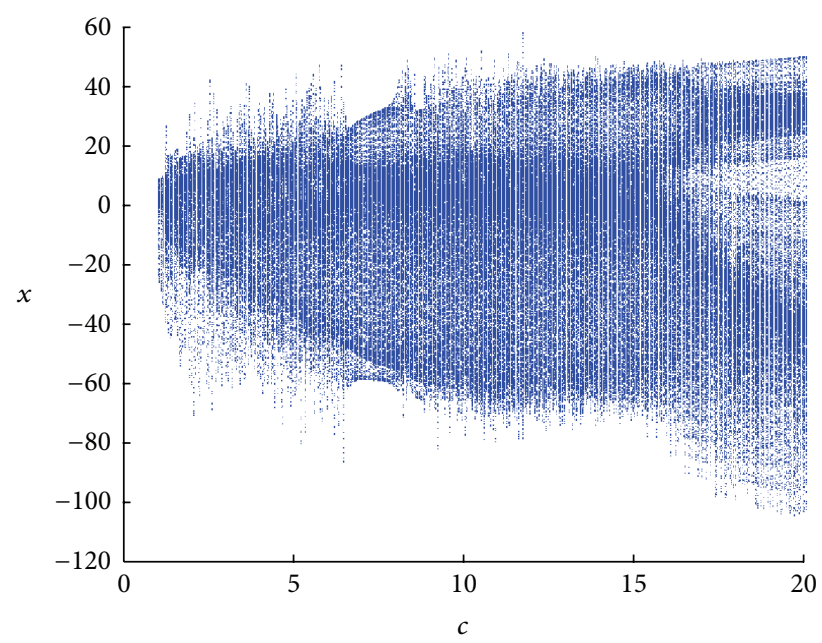

FIgURE 2: Bifurcation diagram in $x$-direction with $c$ varies.

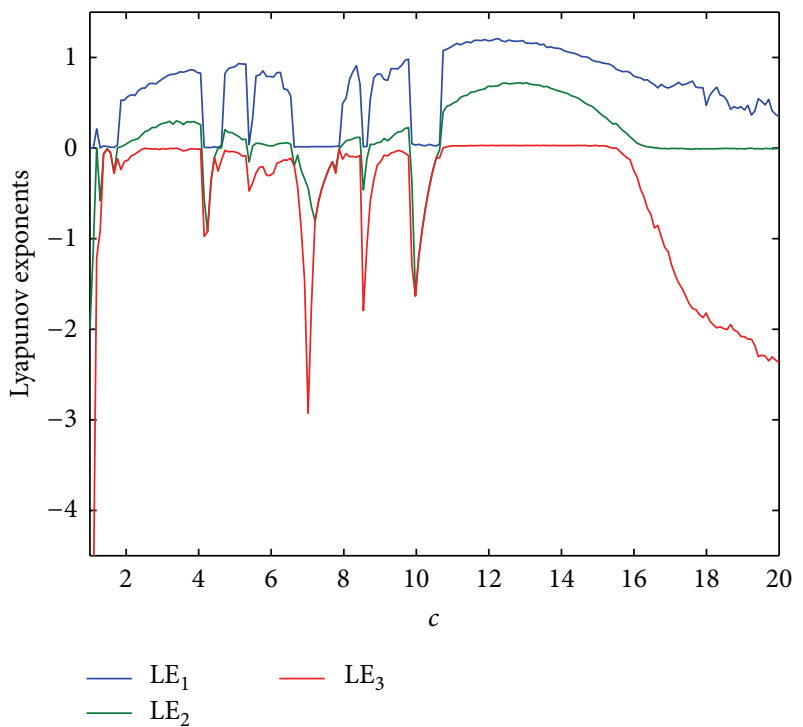

FIgURE 3: Lyapunov exponents $\mathrm{LE}_{1}, \mathrm{LE}_{2}, \mathrm{LE}_{3}$.

$e=y-x$, we choose a terminal sliding mode surface $s=0$, where

$$
s=\dot{e}+\alpha e+\beta e^{q / p},
$$

where $s \in R^{n}, \alpha, \beta$ are diagonal matrixes. $e^{q / p}=\left[e_{1}^{q_{1} / p_{1}}\right.$, $\left.e_{2}^{q_{2} / p_{2}}, \ldots, e_{n}^{q_{n} / p_{n}}\right]^{T}\left(q_{i}, p_{i}\right)$ are parameters and $q_{i}<p_{i}<2 q_{i}$.

Theorem 1. If $\delta(t)$ and $\dot{\delta}(t)$ are bounded, with the above terminal sliding mode surface, one can synchronize two different

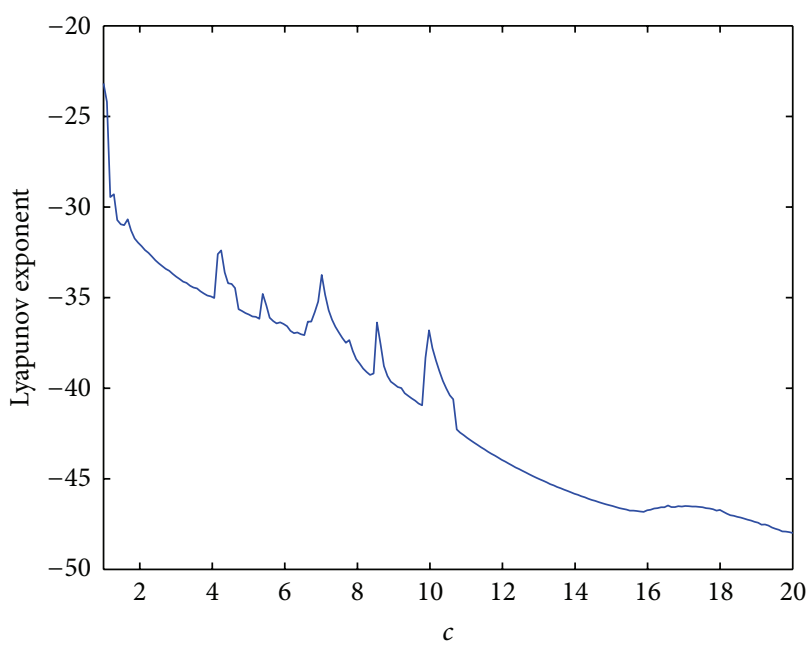

$-\mathrm{LE}_{4}$

FIgURE 4: Lyapunov exponent $\mathrm{LE}_{4}$.

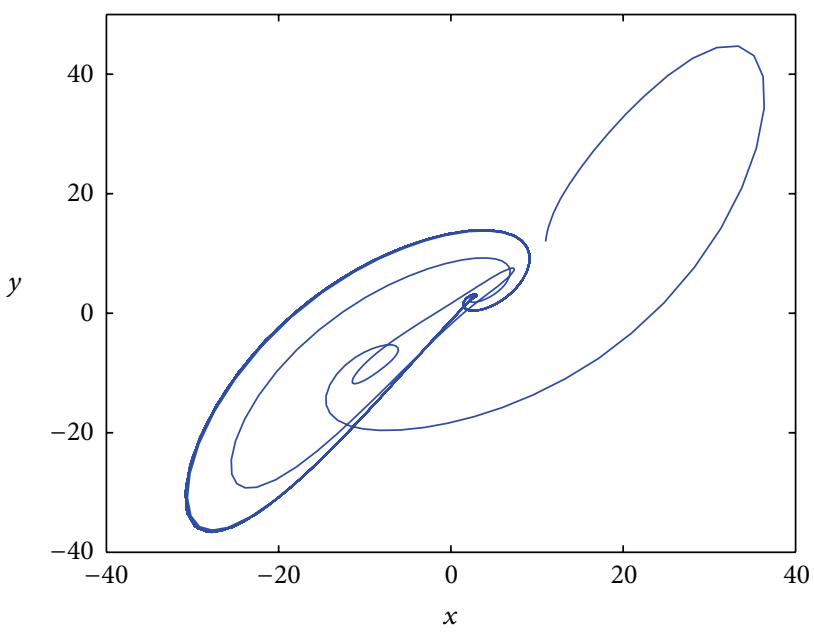

(a)

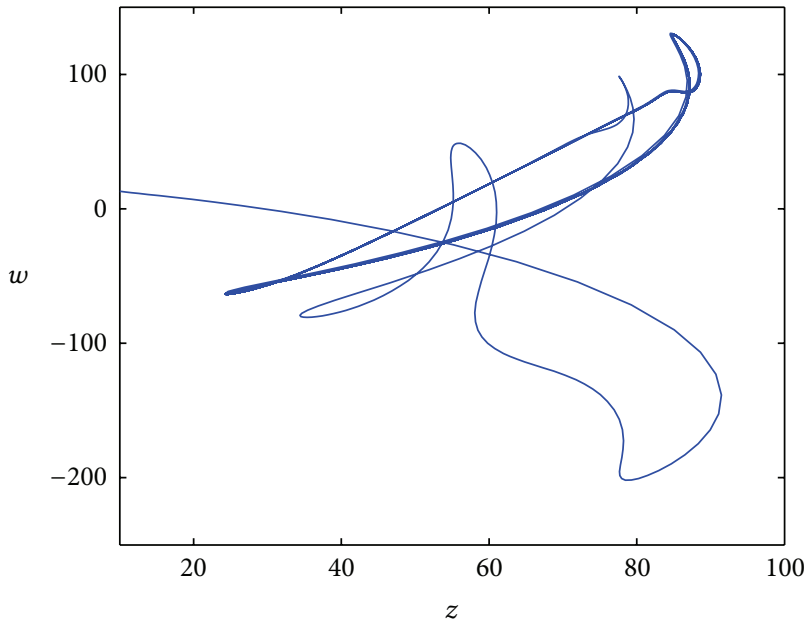

(b)

FIgURE 5: $c=1.1$, periodic attractor. 


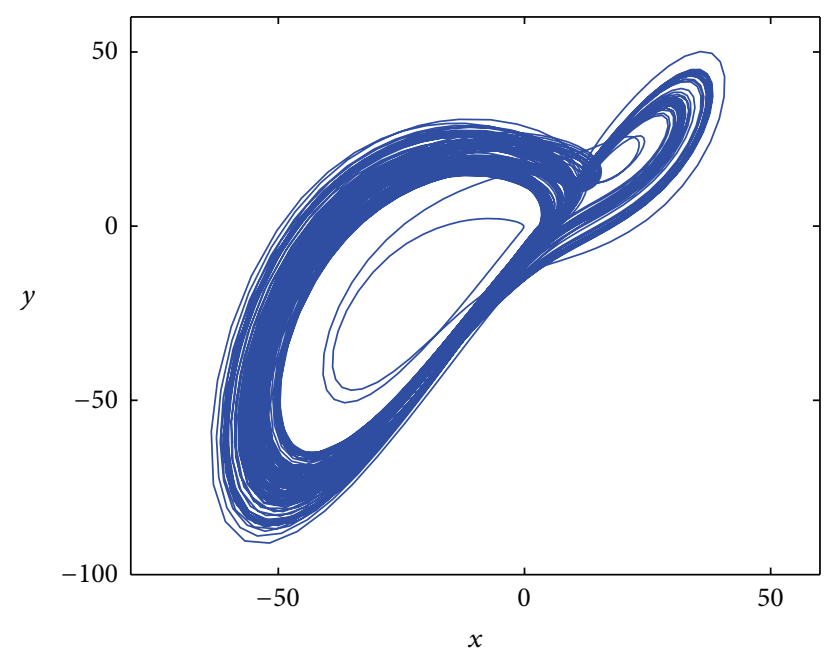

(a)

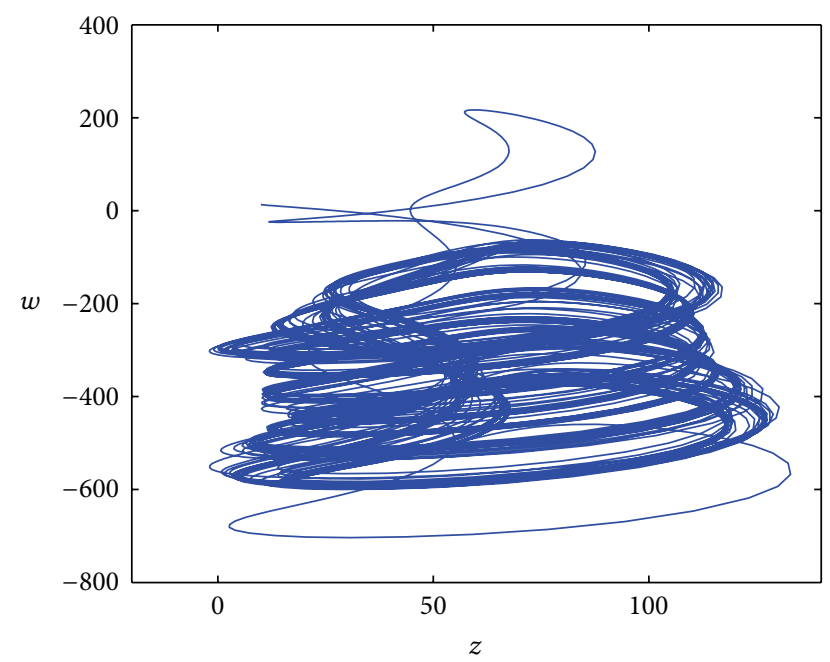

(b)

Figure $6: c=7.87$, quasi-periodic attractor.

chaoses with disturbance by using the terminal sliding mode controller $u$, provided by

$$
\begin{aligned}
\dot{u}+( & \left.\frac{\partial g}{\partial e}+\alpha+\beta \frac{q}{p} e^{q / p-1}\right) u \\
= & -\left(\frac{\partial g}{\partial x}-\frac{\partial f}{\partial x}\right) \dot{x}-\left(\frac{\partial g}{\partial e}+\alpha+\beta \frac{q}{p} e^{q / p-1}\right)(g-f) \\
& -\epsilon s-2^{-\eta} \omega s^{2 \eta-1}-D \operatorname{sign}(s),
\end{aligned}
$$

where $\epsilon, \omega$ are diagonal matrices, $\eta \in R^{n}$ is parameter, and $D>\left|\left(\partial g / \partial e+\alpha+\beta(q / p) e^{q / p-1}\right) \delta(t)+d \delta(t) / d t\right|$.

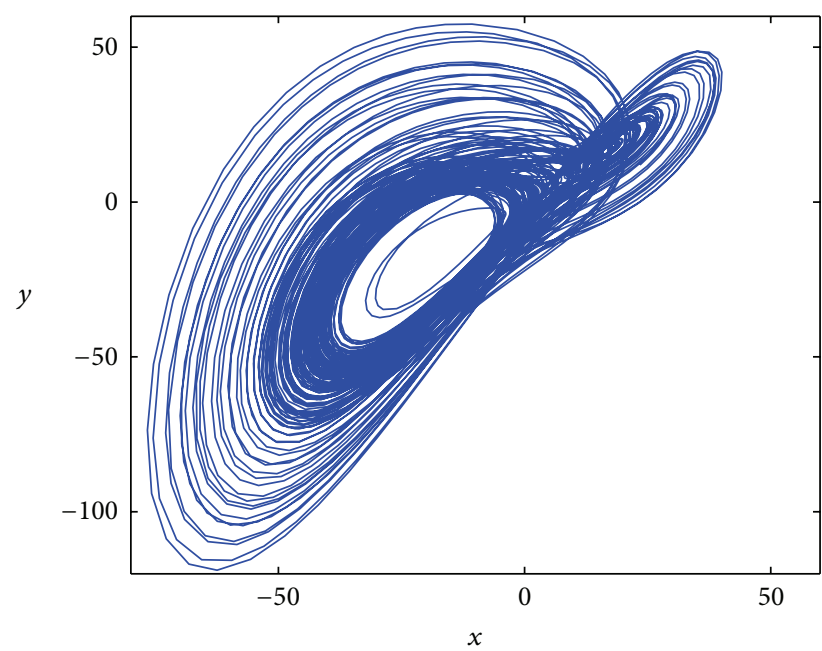

(a)

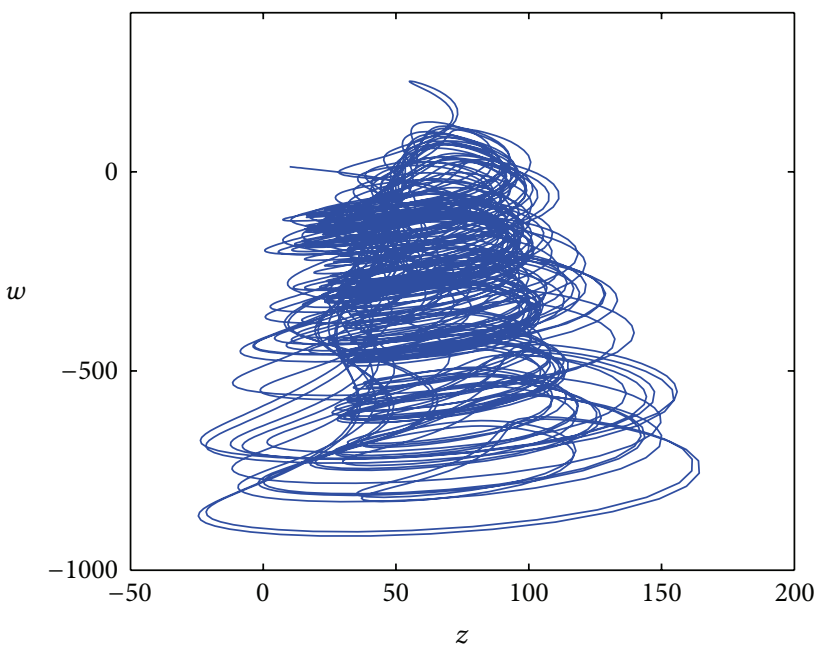

(b)

FIGURE 7: $c=6$, chaotic attractor.

Proof. First, we all know that our design target is to make $s \dot{s}<$ 0 . For the given terminal sliding mode surface $s$,

$$
\begin{aligned}
\dot{s}= & \frac{\partial g}{\partial x} \dot{x}+\frac{\partial g}{\partial e} \dot{e}+\frac{d \delta(t)}{d t}+\dot{u}+\alpha \dot{e}+\beta \frac{q}{p} e^{q / p-1} \dot{e}-\frac{\partial f}{\partial x} \dot{x} \\
= & \left(\frac{\partial g}{\partial x}-\frac{\partial f}{\partial x}\right) \dot{x}+\left(\frac{\partial g}{\partial e}+\alpha+\beta \frac{q}{p} e^{q / p-1}\right)(g-f) \\
& +\left(\frac{\partial g}{\partial e}+\alpha+\beta \frac{q}{p} e^{q / p-1}\right) \delta(t)+\left(\frac{\partial g}{\partial e}+\alpha+\beta \frac{q}{p} e^{q / p-1}\right) u \\
& +\frac{d \delta(t)}{d t}+\dot{u} .
\end{aligned}
$$




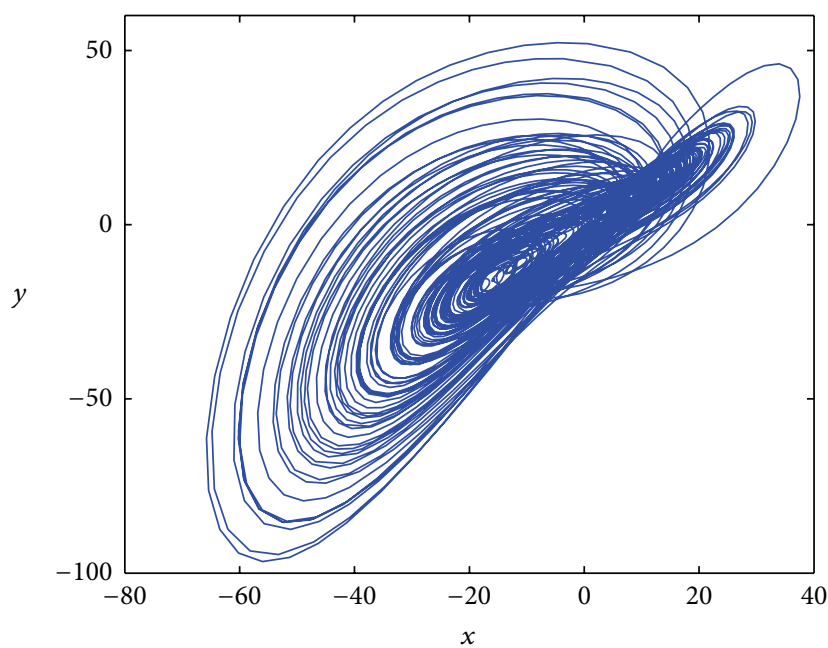

(a)

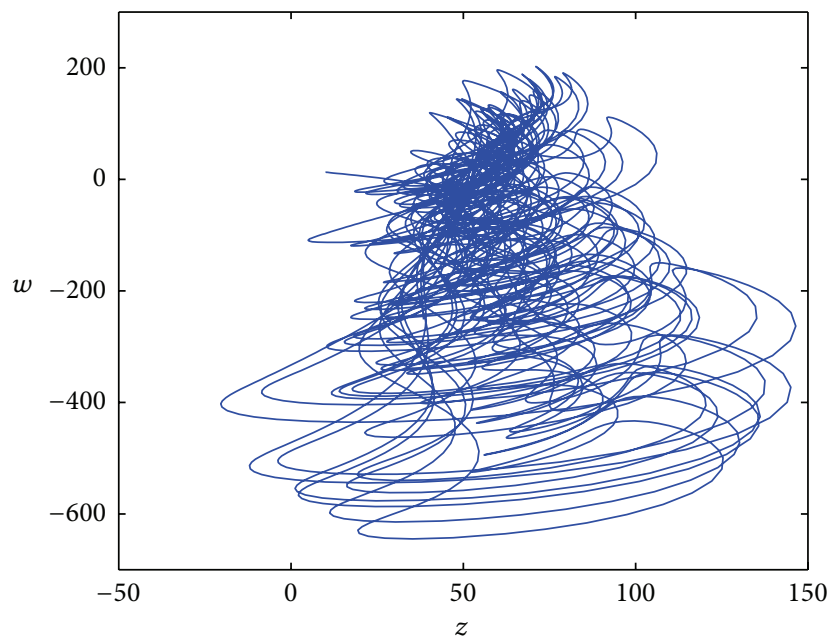

(b)

FIGURE 8: $c=2.8$, hyperchaotic attractor.

If we use the controller above, then,

$$
\begin{aligned}
\dot{s}= & \left(\frac{\partial g}{\partial e}+\alpha+\beta \frac{q}{p} e^{q / p-1}\right) \delta(t)+\frac{d \delta(t)}{d t} \\
& -\epsilon s-2^{-\eta} \omega s^{2 \eta-1}-D \operatorname{sign}(s) .
\end{aligned}
$$

And then we have

$$
\begin{aligned}
s \dot{s}= & \left(\left(\frac{\partial g}{\partial e}+\alpha+\beta \frac{q}{p} e^{q / p-1}\right) \delta(t)+\frac{d \delta(t)}{d t}\right) s-\epsilon s^{2} \\
& -2^{-\eta} \omega s^{2 \eta}-D|s|<-\epsilon s^{2}-2^{-\eta} \omega s^{2 \eta}-D|s| .
\end{aligned}
$$

So with the given controller two different chaoses can be synchronized.

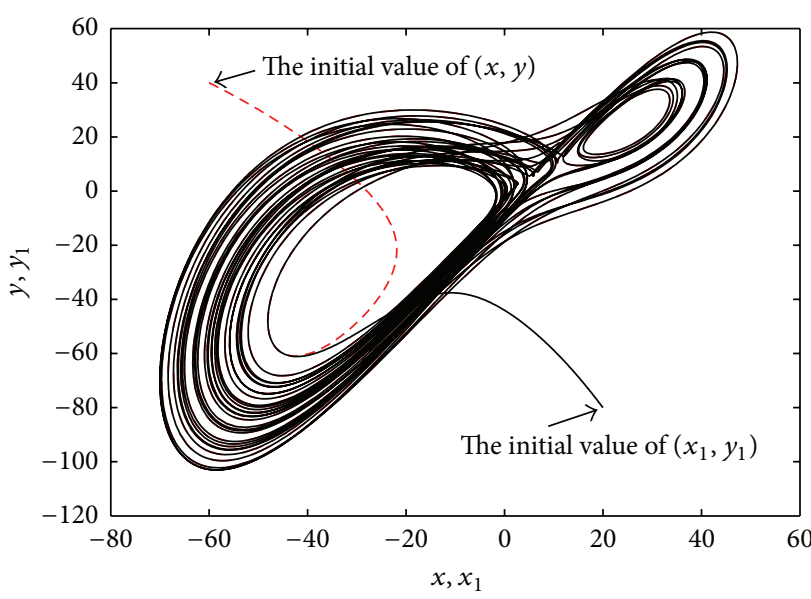

FIGURE 9: The synchronization phase portrait of the new system and hyperchaotic Chen system with disturbance.

From the proof above, we know that switching item $D \operatorname{sign}(s)$ is two-valued; that is, it equals $D$ or $-D$ and it always work. If we let it be a step function, that is,

$$
D^{*}=\left\{\begin{array}{l}
D(\text { a positive constant }), \\
\text { if }\left(\left(\frac{\partial g}{\partial e}+\alpha+\beta \frac{q}{p} e^{q / p-1}\right) \delta(t)\right. \\
\left.\quad+\frac{d \delta(t)}{d t}\right) s-\epsilon s^{2}-2^{-\eta} \omega s^{2 \eta}>0 \\
0, \quad \text { otherwise, }
\end{array}\right.
$$

or use saturation function. These schemes can attenuate the chattering of the sliding mode surface. Also we know the item $-\epsilon s-2^{-\eta} \omega s^{2 \eta-1}$ guarantees that the state trajectory reaches the sliding mode surface in a short time.

\section{Numerical Simulation}

Here, we assume that the new system introduced in this paper is the drive system and hyperchaotic Chen system, is the response system:

$$
\begin{gathered}
\dot{x}_{1}=a_{1}\left(-x_{1}+y_{1}\right)+w_{1}, \\
\dot{y}_{1}=b_{1} x_{1}-x_{1} z_{1}+c_{1} y_{1}, \\
\dot{z}_{1}=x_{1} y_{1}-d_{1} z_{1}, \\
\dot{w}_{1}=y_{1} z_{1}+n_{1} w_{1},
\end{gathered}
$$

where $x_{1}, y_{1}, z_{1}$, and $w_{1}$ are variables of hyperchaotic Chen system and $a_{1}, b_{1}, c_{1}, d_{1}$, and $n_{1}$ are parameters. Adding controllers and noise perturbations to the response system, we get

$$
\begin{gathered}
\dot{x}_{1}=a_{1}\left(-x_{1}+y_{1}\right)+w_{1}+d_{1}+u_{1}, \\
\dot{y}_{1}=b_{1} x_{1}-x_{1} z_{1}+c_{1} y_{1}+d_{2}+u_{2}, \\
\dot{z}_{1}=x_{1} y_{1}-d_{1} z_{1}+d_{3}+u_{3}, \\
\dot{w}_{1}=y_{1} z_{1}+n_{1} w_{1}+d_{4}+u_{4},
\end{gathered}
$$




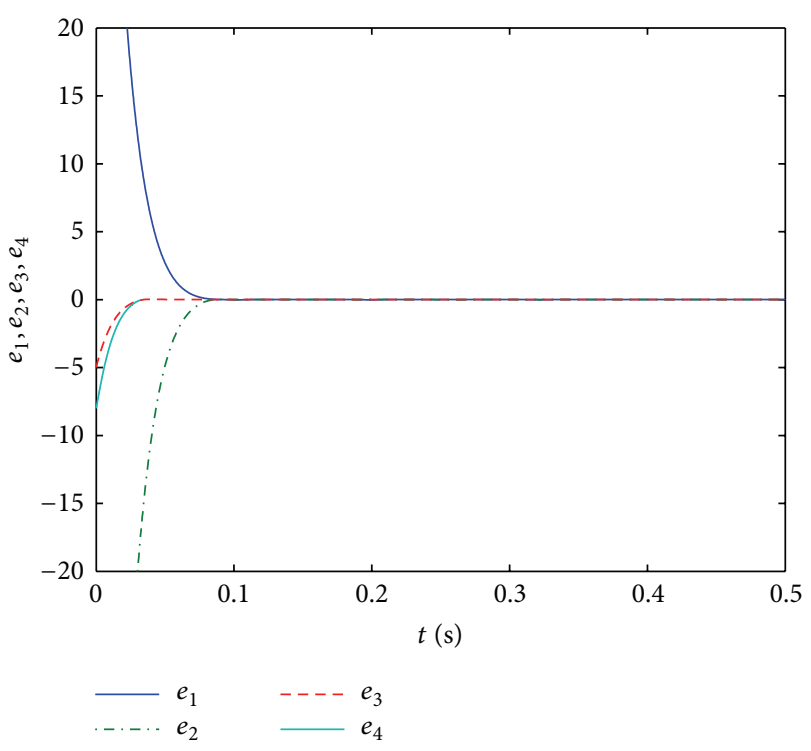

Figure 10: State errors $e_{1}, e_{2}, e_{3}$, and $e_{4}$.

where $u_{1}, u_{2}, u_{3}$, and $u_{4}$ are controllers, $d_{1}, d_{2}, d_{3}$, and $d_{4}$ are disturbances. The state errors are $e_{1}=x_{1}-x, e_{2}=y_{1}-y$, $e_{3}=z_{1}-z$, and $e_{4}=w_{1}-w$.

The sliding surface is

$$
s=\dot{e}+\alpha e+\beta e^{q / p},
$$

where $s=\left[s_{1}, s_{2}, s_{3}, s_{4}\right]^{T}, \dot{e}=\left[\dot{e}_{1}, \dot{e}_{2}, \dot{e}_{3}, \dot{e}_{4}\right]^{T} e=\left[e_{1}, e_{2}, e_{3}\right.$, $\left.e_{4}\right]^{T}, \alpha=\operatorname{diag}\left(\alpha_{1}, \alpha_{2}, \alpha_{3}, \alpha_{4}\right), \beta=\operatorname{diag}\left(\beta_{1}, \beta_{2}, \beta_{3}, \beta_{4}\right), e^{q / p}=$ $\left[e_{1}^{q_{1} / p_{1}}, e_{2}^{q_{2} / p_{2}}, e_{3}^{q_{3} / p_{3}}, e_{4}^{q_{4} / p_{4}}\right]^{T}, p_{i}<q_{i}<2 p_{i}, 1 \leq i \leq 4$.

To demonstrate the effectiveness of the controller, we give the parameters and initial value of the drive system as $a=$ $31, b=54, c=13, d=1.8$ and $x_{0}=[-60,40,8,10]$. And the parameters and initial value of the response system are given as $a_{1}=35, b_{1}=7, c_{1}=12, d_{1}=3, n_{1}=0.7$ and $x_{0}^{*}=[20,-80,3,2]$ while the disturbaces are chosen as $\delta_{1}=$ $0.025 \sin (t), \delta_{2}=0.05 \cos (t), \delta_{3}=\delta_{4}=0.5$. But the cotroller parameters are the following $\alpha_{1}=50, \alpha_{2}=48, \alpha_{3}=50$, $\alpha_{4}=51, \beta_{1}=50, \beta_{2}=52, \beta_{3}=53, \beta_{4}=62, q_{1}=3, q_{2}=3$, $p_{2}=5, p_{4}=5, p_{3}=5, p_{1}=5, q_{4}=3, q_{3}=3, \varepsilon_{1}=30$, $\varepsilon_{2}=30, \varepsilon_{3}=30, \varepsilon_{4}=15, \omega_{1}=10, \omega_{2}=10, \omega_{3}=10$, $\omega_{4}=7, \eta=2$ and $D=1$. By the way, the diagrams of the synchronized states, the state errors and the controllers of the new system and hyperchaotic Chen system are presented as Figures 9, 10, and 11 .

From the above diagrams, we know that the precision synchronization of the two different systems with disturbance can be realized by less than 0.1 seconds. Synchronization time can change by tuning parameters.

\section{Conclusion}

In this paper, we have introduced a new hyperchaotic system by adding a state variable $w$ and investigated the characters of the system. By using fast terminal sliding mode control scheme, the synchronization of two different chaotic systems

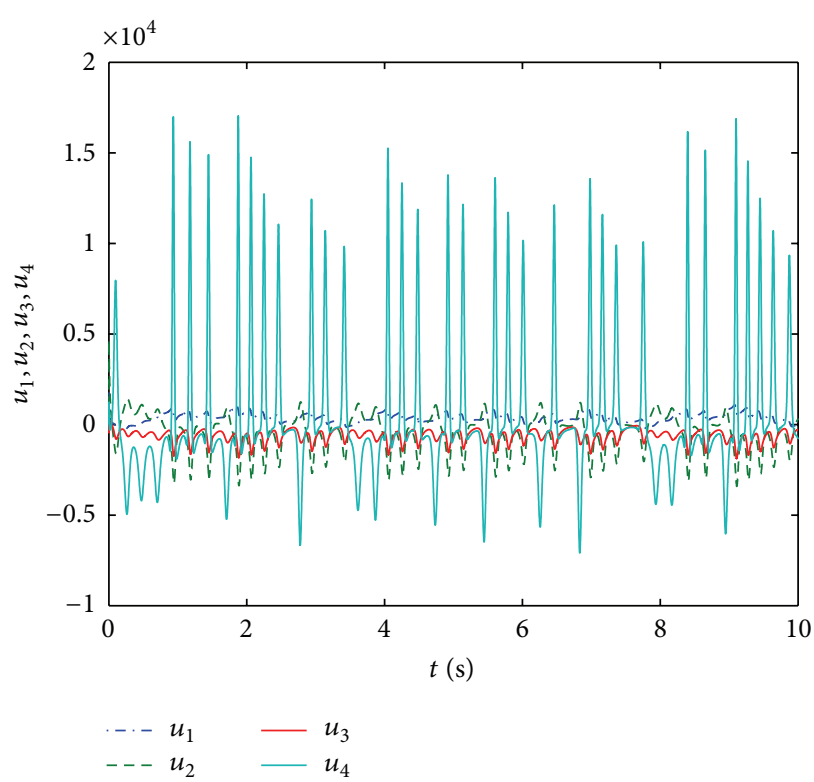

FIGURE 11: The controllers $u_{1}, u_{2}, u_{3}, u_{4}$.

is studied. Synchronization of the new hyperchaotic system and hyperchaotic Chen system with disturbance has illustrated effectiveness of the scheme.

\section{Acknowledgments}

This work is supported by National Natural Science Foundation (NNSF) of China under Grant no. 61374118 and Youth Foundation of Qujing Normal University (2008QN034).

\section{References}

[1] E. N. Lorenz, "Deterministic non-periodic flow," Journal of the Atmospheric Sciences, vol. 20, pp. 130-141, 1963.

[2] O. E. Rossler, "An equation for continuous chaos," Physics Letters A, vol. 57, no. 5, pp. 397-398, 1976.

[3] R. Genesio and A. Tesi, "Harmonic balance methods for the analysis of chaotic dynamics in nonlinear systems," Automatica, vol. 28, no. 3, pp. 531-548, 1992.

[4] G. Chen and T. Ueta, "Yet another chaotic attractor," International Journal of Bifurcation and Chaos, vol. 9, no. 7, pp. 14651466, 1999.

[5] J. Lü and G. Chen, "A new chaotic attractor coined," International Journal of Bifurcation and Chaos in Applied Sciences and Engineering, vol. 12, no. 3, pp. 659-661, 2002.

[6] G. R. Chen and J. H. Lü, Dynamics of the Lorenz System Family: Analysis, Control and Synchronization, Science Press, Beijing, China, 2003, Chinese.

[7] C. Liu, T. Liu, L. Liu, and K. Liu, "A new chaotic attractor," Chaos, Solitons \& Fractals, vol. 22, no. 5, pp. 1031-1038, 2004.

[8] G. Qi, S. Du, G. Chen, Z. Chen, and Z. Yuan, "On a fourdimensional chaotic system," Chaos, Solitons \& Fractals, vol. 23, no. 5, pp. 1671-1682, 2005.

[9] O. E. Rossler, "An equation for hyperchaos," Physics Letters A, vol. 71, no. 2-3, pp. 155-157, 1979. 
[10] G. R. Chen and X. Dong, From Chaos to Order: Methodologies, Perspectives and Applications, World Scientific, Singapore, 1998.

[11] Y. Li, W. K. S. Tang, and G. Chen, "Generating hyperchaos via state feedback control," International Journal of Bifurcation and Chaos in Applied Sciences and Engineering, vol. 15, no. 10, pp. 3367-3375, 2005.

[12] A. Chen, J. Lu, J. Lü, and S. Yu, "Generating hyperchaotic Lü attractor via state feedback control," Physica A, vol. 364, pp. 103110, 2006

[13] S. Nikolov and S. Clodong, "Occurrence of regular, chaotic and hyperchaotic behavior in a family of modified Rossler hyperchaotic systems," Chaos, Solitons \& Fractals, vol. 22, no. 2, pp. 407-431, 2004.

[14] X. Wang and M. Wang, "A hyperchaos generated from Lorenz system," Physica A, vol. 387, no. 14, pp. 3751-3758, 2008.

[15] R. Barboza, "Dynamics of a hyperchaotic Lorenz system," International Journal of Bifurcation and Chaos, vol. 17, no. 12, pp. 4285-4294, 2007.

[16] L. M. Pecora and T. L. Carroll, "Synchronization in chaotic systems," Physical Review Letters, vol. 64, no. 8, pp. 821-824, 1990.

[17] M.-C. Ho and Y.-C. Hung, "Synchronization of two different systems by using generalized active control," Physics Letters A, vol. 301, no. 5-6, pp. 424-428, 2002.

[18] L. Huang, R. Feng, and M. Wang, "Synchronization of chaotic systems via nonlinear control," Physics Letters A, vol. 320, no. 4, pp. 271-275, 2004.

[19] T.-L. Liao, "Adaptive synchronization of two Lorenz systems," Chaos, Solitons \& Fractals, vol. 9, no. 9, pp. 1555-1561, 1998.

[20] S. Chen and J. Lü, "Synchronization of an uncertain unified chaotic system via adaptive control," Chaos, Solitons \& Fractals, vol. 14, no. 4, pp. 643-647, 2002.

[21] Z. Li, C. Han, and S. Shi, "Modification for synchronization of Rossler and Chen chaotic systems," Physics Letters A, vol. 301, no. 3-4, pp. 224-230, 2002.

[22] E.-W. Bai and K. E. Lonngren, "Sequential synchronization of two Lorenz systems using active control," Chaos, Solitons \& Fractals, vol. 11, no. 7, pp. 1041-1044, 2000.

[23] M. Yahyazadeh, A. Ranjbar Noei, and R. Ghaderi, "Synchronization of chaotic systems with known and unknown parameters using a modified active sliding mode control," ISA Transactions, vol. 50, no. 2, pp. 262-267, 2011.

[24] H.-T. Yau, "Chaos synchronization of two uncertain chaotic nonlinear gyros using fuzzy sliding mode control," Mechanical Systems and Signal Processing, vol. 22, no. 2, pp. 408-418, 2008.

[25] X. Yu and Y. Wu, "Adaptive terminal sliding mode control of uncertain nonlinear systems-backstepping approach," Control Theory and Applications, vol. 15, no. 6, pp. 900-907, 1998.

[26] N. N. Zhang, The Theory and Application of Terminal Sliding Mode Control, Science Press, Beijing, China, 2011. 


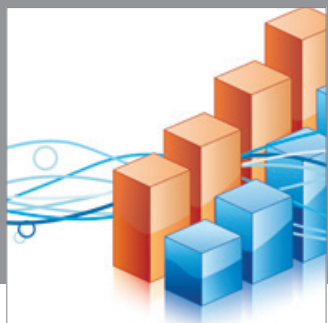

Advances in

Operations Research

mansans

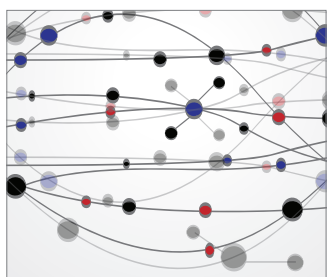

The Scientific World Journal
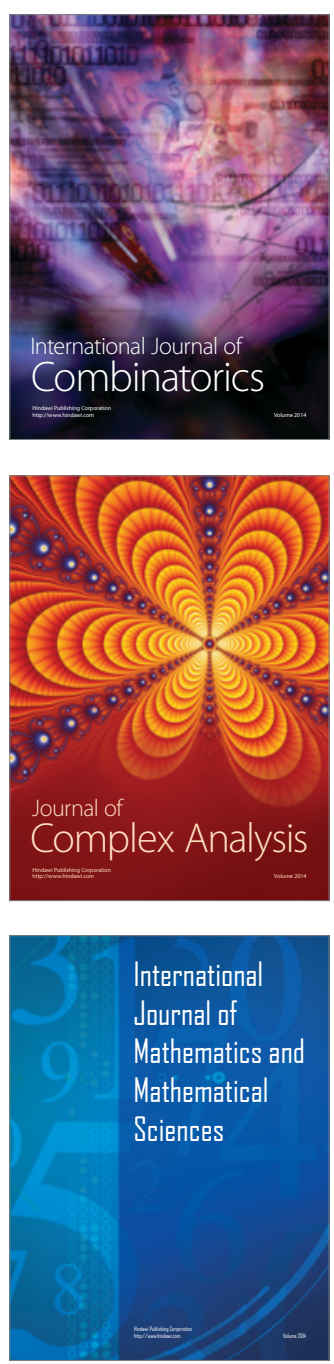
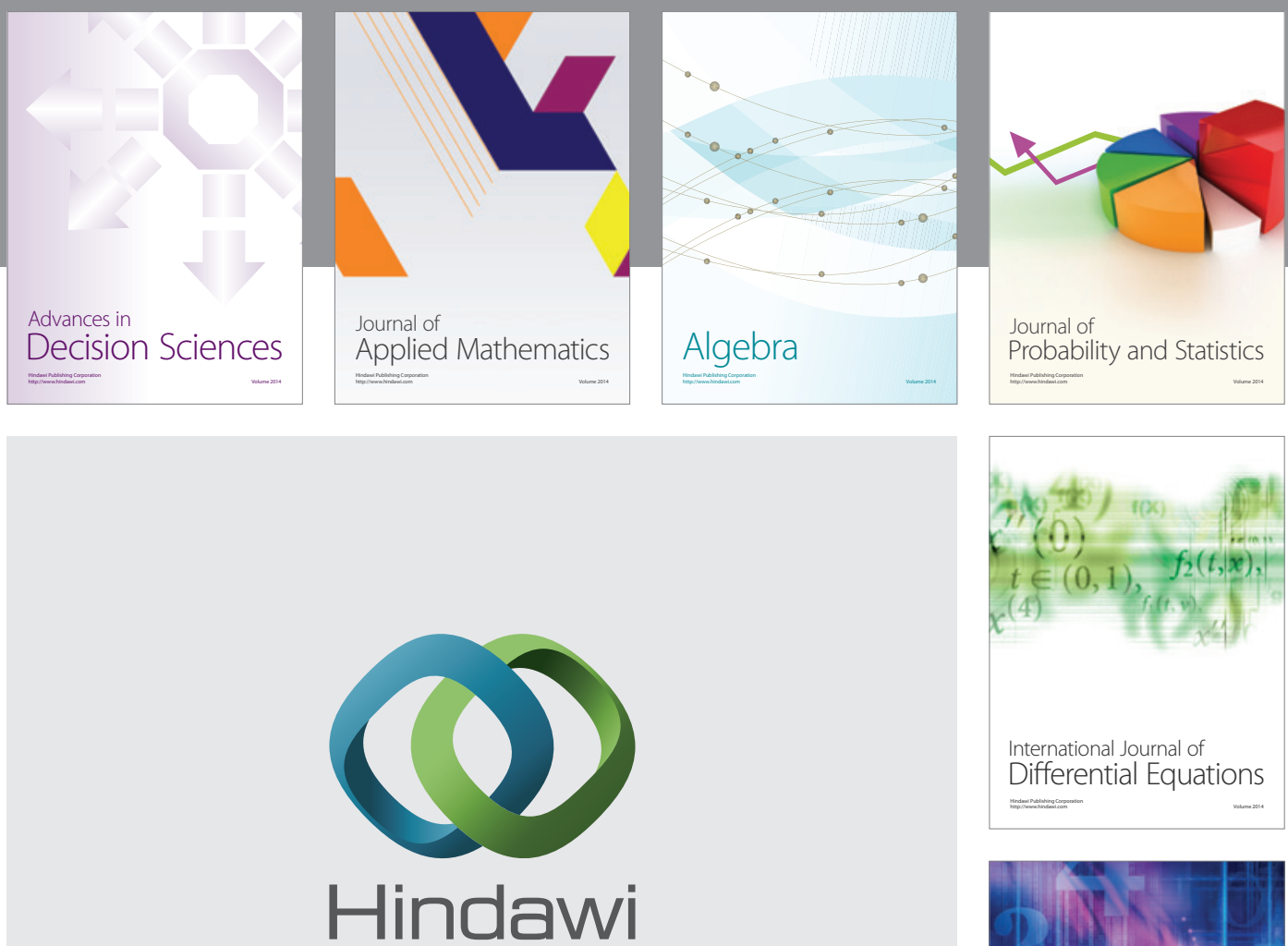

Submit your manuscripts at http://www.hindawi.com
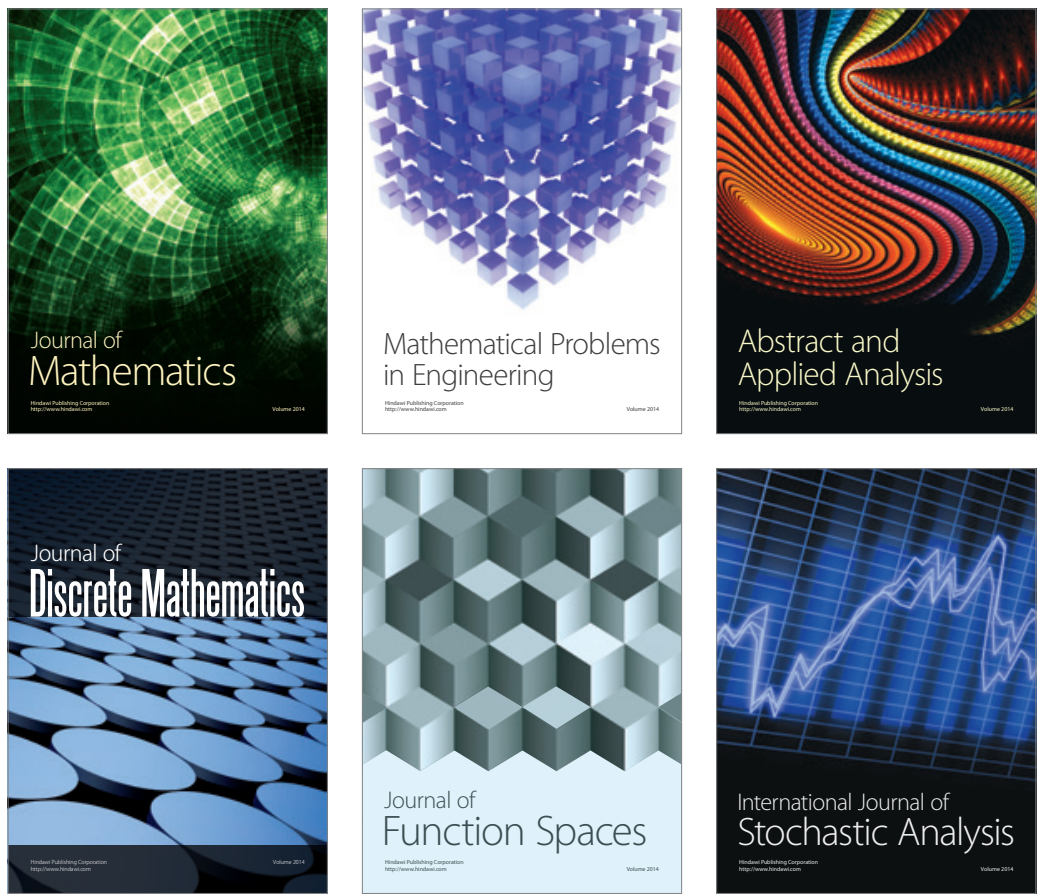

Journal of

Function Spaces

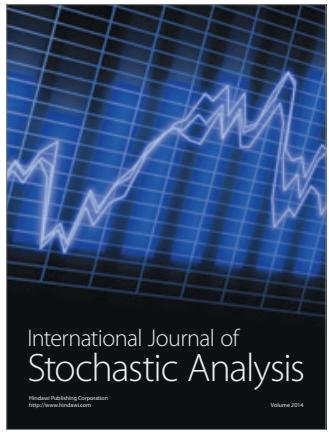

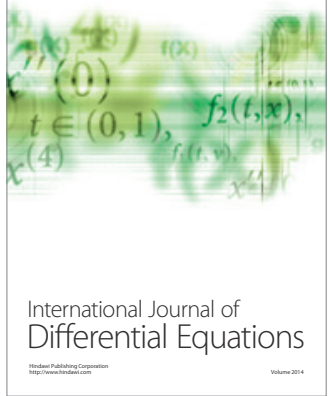
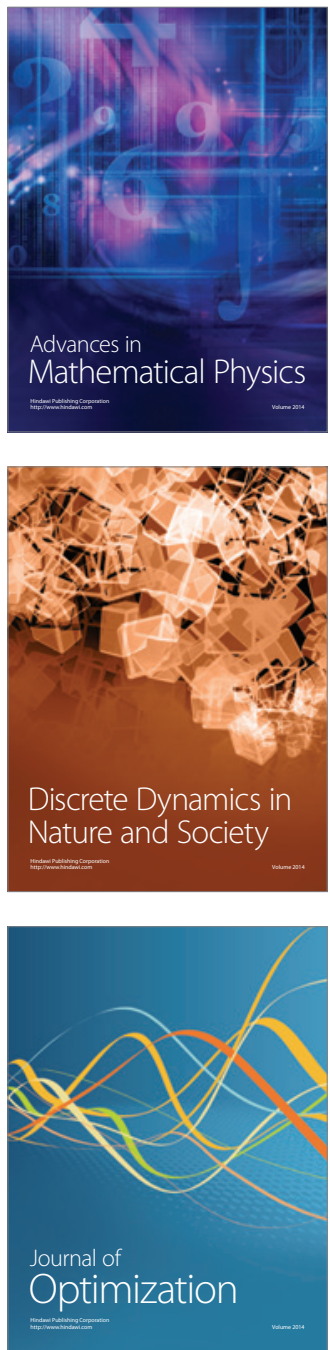